AN. MED INTERNA (Madrid) AN. MED. INTERNA (Madrid)
Vol. 20, N. 1 , pp. 31-33, 2003

\title{
Paniculitis mesentérica con afectación retroperitoneal resuelta tras tratamiento con pulsos de ciclofosfamida endovenosa
}

\author{
E. COLOMER RUBIO, A. BLANES GALLEGO, C. CARBONELL BIOT, A. VILLAR \\ GRIMALT, H. TOMÁS IVORRA, A. LLAMUSÍ LORENTE
}

Servicio de Medicina Interna. Hospital Arnau de Vilanova. Valencia

\begin{abstract}
RESUMEN
La paniculitis mesentérica es un proceso inflamatorio del tejido graso mesentérico. Cursa con un engrosamiento del mesenterio del intestino delgado o colon y puede afectar en ocasiones al retroperitoneo. Se caracteriza por una infiltración de macrófagos cargados de grasa y asociados a un grado variable de inflamación y fibrosis. En el tratamiento se ha empleado colchicina, dapsona, corticoides asociados o no a inmunosupresores, pero no hay estudios controlados prospectivos que definan el tratamiento adecuado. Además hay casos de regresión sin terapéutica específica.

Presentamos el caso de una paciente con paniculitis mesentérica con afectación del colon y espacio retroperitoneal englobando el uréter y la vena ilíaca derechos. El tratamiento médico inmunosupresor con ciclofosfamida en pulsos mensuales endovenosos y corticoides orales, consiguió la desaparición de la masa abdominal sin recaída durante diez meses de seguimiento.
\end{abstract}

PALABRAS CLAVE: Paniculitis mesentérica. Retroperitoneo. Ciclofosfamida.
MESENTERIC PANNICULITIS WITH RETROPERITONEAL INVOLVE MENT RESOLVED AFTER TREATMENT WITH INTRAVENOUS CYCLOPHOSPHAMIDE PULSES

\begin{abstract}
Mesenteric panniculitis is an inflammatory process of the adipose tissue of the mesentery. It produces a thickening of the mesentery of the small bowel or colon and can occasionally involve the retroperitoneum. It is characterized by an infiltration of lipid-laden macrophages and associated with variable degrees of inflammation and fibrosis. Several treatments have been used, including colchicine, dapsone, corticoste roids associated or not with immunosupresants, but there are no pros pective controlled studies to define appropiate treatment; moreover, the re are cases of regression without specific therapy.

We present the case of a patient with mesenteric panniculitis afecting the colon and retroperitoneal space including the right ureter and iliac vein. Immunosuppresive treatment with monthly intravenous pulse cyclop hosphamide and oral corticosteroids, resulted in the disappearance of the abdominal mass without relapse during ten months of monitoring.
\end{abstract}

KEY WORDS: Mesenteric panniculitis. Retroperitoneum. Cyclophopha mide.

Colomer Rubio E, Blanes Gallego A, Carbonell Biot C, Villar Grimalt A, Tomás Ivorra H, Llamusí Lorente A. Paniculitis mesentérica con afectación retroperitoneal resuelta tras tratamiento con pulsos de ciclofosfamida endovenosa. An Med Interna (Madrid) 2003; 20: 31-33.

\section{INTRODUCCIÓN}

La paniculitis mesentérica es un proceso inflamatorio idiopático poco frecuente, que afecta al tejido graso del mesenterio, engrosándolo. Afecta fundamentalmente al mesenterio del intestino delgado (1). La etiología es desconocida, aunque se han apuntado como posibles agentes causales: la isquemia, infecciones, traumatismos abdominales (como la vibración contínua de un martillo neumático) (2), antecedentes quirúrgicos, fármacos y fenómenos autoinmunes (1). Afecta con más frecuencia a hombres entre 50 y 70 años, en una proporción por sexos de 2-3:1 (4).

Presentamos a continuación, un caso de una paciente con paniculitis mesentérica, con afectación de colon y retroperitoneo que englobaba el uréter y vena ilíaca derechos. Con tratamiento médico inmunosupresor de ciclofosfamida endovenosa en pulsos mensuales y corticoides, se consiguió una rápida mejoría clínica con desaparición de la masa abdominal y sin recidiva durante los diez meses de seguimiento.

\section{CASO APORTADO}

Mujer de 79 años, con antecedentes de hipertensión arterial en tratamiento con nifedipino, que acudió al servicio de urgencias por dolor y edema en miembro inferior derecho de doce horas de evolución. Presentaba desde hacía dos meses cuadro de diarrea líquida, sin productos patológicos, acompañado de astenia, anorexia y pérdida de peso no cuantificada.

Examen físico: Afebril. Tensión arterial 125/75 mmHg. Regular estado general, discreta palidez cutánea. Auscultación cardíaca y pulmonar, sin alteraciones. Abdomen blando y depresible, sin signos de irritación peritoneal, ni puntos dolorosos. En fosa ilíaca derecha, se palpaba una masa de límites irregulares, de consistencia dura, adherida a planos profundos y no dolorosa a la palpación. Puño-percusión renal bilateral negativa. En extremidad inferior derecha, edema desde la raíz del muslo y aumento de la temperatura local, dolor a la palpación del gemelo y no se apreciaban cordones venosos. Pulsos periféricos presentes y simétricos.

Datos de laboratorio: destacan hematocrito 30,3\%, hemoglobina 9,8 g/dl, VCM 82,1 fl, hierro sérico $101 \mathrm{mcg} / \mathrm{dl}$, ferritina $492 \mathrm{mg} / \mathrm{dl}$, LDH $771 \mathrm{mU} / \mathrm{ml}$. Fórmula y recuento, plaquetas y coagulación normales. Serología de VIH y hepatitis víricas negativas. Resto de parámetros bioquímicos habituales, incluyendo orina elemental y sedimen-

Trabajo aceptado: 23 de octubre de 2001

Correspondencia: Enrique Colomer Rubio. Ramón Llull, 31, puerta 14. 46021 Valencia. 
to, sin alteraciones. Prueba de Mantoux negativa. Pruebas complementarias : Rx tórax: sin alteraciones. Eco-doppler venosa de extremidad inferior derecha: trombosis venosa profunda que afectaba a vena femoral común, superficial y poplítea profunda derechas. Enema opaco : estenosis a nivel del sigma. Tránsito intestinal: en pelvis menor masa que no dependía de forma intrínseca de las asas ileales, las cuales estaban comprimidas y desplazadas. Colonoscopia: mucosa edematosa a 20-40 cm del ano, que colapsaba la luz, se tomaron biopsias informadas como inflamación crónica inespecífica. TAC abdómino pélvico (Fig. 1): uropatía obstructiva de riñón derecho, secundaria a atrapamiento del uréter por masa localizada a nivel del mesogastrio y fosa ilíaca derecha, que englobaba asas de intestino delgado y también el polo cecal. Se observaba marcado engrosamiento de la grasa mesentérica y del mesosigma. Trombosis de ilíaca y femorales derechas.

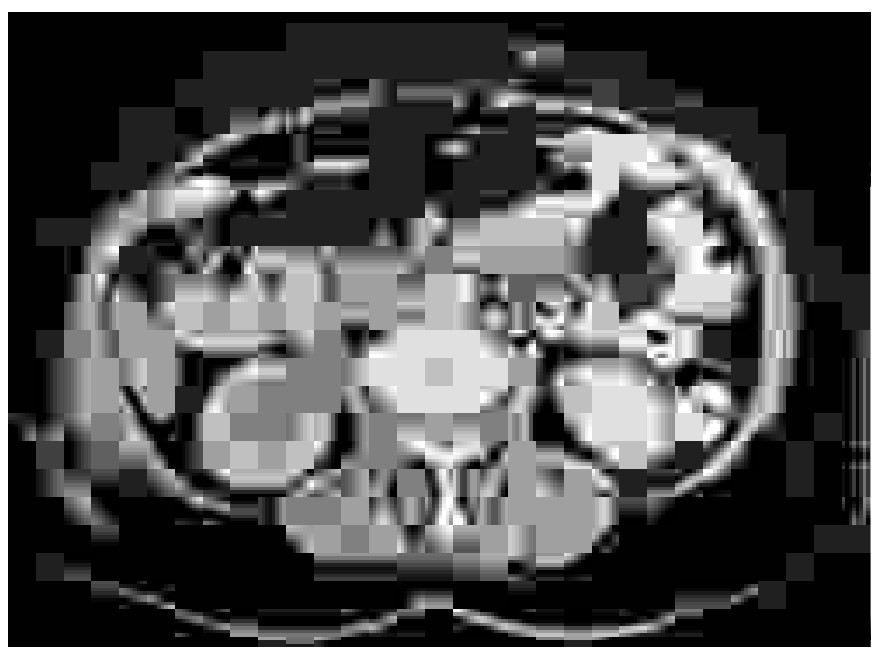

Fig. 1. TAC abdómino-pélvico. Hidronefrosis riñón derecho. M arcado engrosamiento de la grase mesentérica que engloba y desplaza las asas de intestino delgado.

Biopsia de médula ósea : normocelular, moderada diseritropoyesis. No se observan células linfoides atípicas. Cultivo, tinción de Ziehl-Neelsen y Lowestein de médula ósea negativos.

$\mathrm{Al}$ ingreso se inició tratamiento con heparina de bajo peso molecular a dosis anticoagulante. Ante el resultado de las exploraciones se decidió realizar laparotomía que objetivó gran masa mesentérica y retroperitoneal de consistencia pétrea, que englobaba uréter derecho, intestino delgado y colon descendente. Se tomaron múltiples biopsias con el resultado de proliferación miofibroblástica con abundantes depósitos de colágeno hialino. En zonas adyacentes a éstas y en transición con ellas se observó necrosis grasa con presencia de numerosos lipófagos y densos infiltrados inflamatorios, de carácter agudo en unas ocasiones y de predominio linfoplasmocitario en otras, todo ello compatible con paniculitis mesentérica. También se remitieron muestras para cultivo ordinario y de micobacterias y tinción de PAS, que fueron negativos.

Se inició tratamiento con ciclofosfamida en pulsos mensuales endovenosos a dosis de $0,75 \mathrm{~g} / \mathrm{m}^{2}$ asociado a prednisona oral 1 $\mathrm{mg} / \mathrm{kg}$ de peso con reducción progresiva de la dosis de prednisona y anticoagulación oral con acenocumarol.

La paciente presentó mejoría de su estado general y tras el tercer ciclo con ciclofosfamida, no se palpaba la masa abdominal y no habían signos clínicos de trombosis venosa profunda. Se realizó ecografía abdominal y Eco-doppler venosa de pierna derecha, que no objetivaron signos de uropatía obstructiva ni trombosis venosa.

Tras el cuarto ciclo se solicitó un TAC abdómino-pélvico (Fig. 2), en el que habían desaparecido tanto la masa abdominal como la uropatía obstructiva derecha. Se suspendió la anticoagulación, la prednisona se redujo hasta $5 \mathrm{mg}$ vía oral a días alternos y se completaron hasta 8 ciclos mensuales de tratamiento tras los que se suspendió la ciclofosfamida y la prednisona.

La paciente ha permanecido asintomática durante los diez meses posteriores al tratamiento.

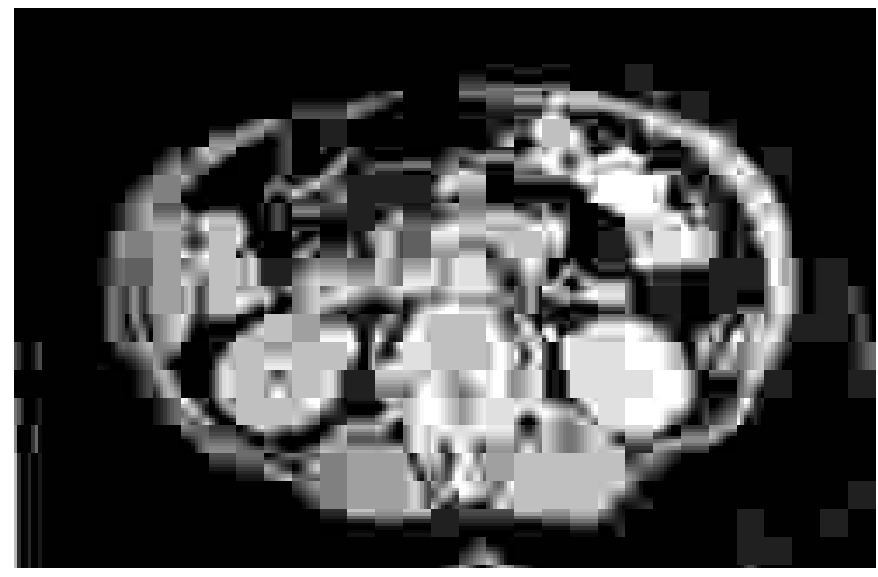

Fig. 2. TAC Abdómino-pélvico de control. Ausencia de uropatía obstructiva y de engrosamiento mesentérico.

\section{DISCUSIÓN}

La paniculitis mesentérica fue descrita por primera vez por Ogden en 1960 (3). Se puede considerar como un estadío de una enfermedad mesentérica poco frecuente $(5,6)$. Al principio aparece lipodistrofia mesentérica, no hay signos de inflamación, el paciente está asintomático y el tratamiento no está indicado. El segundo estadío es la paniculitis mesentérica, microscópicamente se observan macrófagos cargados de lípidos con áreas de necrosis focal e infiltrados inflamatorios. El tercer estadío es el de mesenteritis retráctil en el que destaca la fibrosis. Esta evolución sólo se ha demostrado con el seguimiento de dos casos (1), y por ello en la mayoría de la literatura se considera a la paniculitis mesentérica como una entidad aislada.

La clínica es inespecífica: dolor abdominal (7), fiebre (8), pérdida de peso $(9)$, suboclusión intestinal $(6,10)$ siendo infrecuente la obstrucción completa (11). En la mitad de los pacientes se palpa una masa abdominal mal definida.

La radiología simple no suele aportar datos, y en el estudio de tránsito baritado y enema opaco, el desplazamiento de asas por un efecto masa es el hallazgo más frecuente, como en nuestro caso $(12,13)$. En la TAC $(14,15)$ pueden observarse una o varias masas heterogéneas localizadas en el mesenterio, con densidad diferente según el estadío de la enfermedad y el predominio de inflamación, fibrosis, o grasa. Rodean a los vasos mesentéricos y desplazan las asas intestinales sin invasión de estructuras, lo que la diferencia de la fibrosis retroperitoneal (que sí es invasiva).

Para el diagnóstico definitivo, se ha de descartar la existencia de pancreatitis, enfermedad inflamatoria intestinal o procesos extrabdominales con necrosis grasa (v.g. Enfermedad de WeberChristian), además de la realización de biopsias múltiples. Estas se pueden realizar mediante laparotomía, laparoscopia (16) o a través de biopsia percutánea con aguja gruesa y control por TAC (17). El diagnóstico diferencial, incluye el liposarcoma, linfangioma, sarcoma y carcinoma metastásico que pueden dar imágenes radiológicas similares. Otras patologías a diferenciar son el linfoma esclerosante, los tumores desmoides, la lipomatosis pélvica, el seudotumor inflamatorio o tumor miofibroblástico inflamatorio (18), la enfermedad de Whipple y la fibrosis retroperitoneal, sobre todo cuando la paniculitis alcanza el retroperitoneo como ocurrió en nuestro caso (19). También hay que descartar enfermedades infecciosas por micobacterias (20). La evolución es muy variada, desde pacientes asintomáticos o con episodios repetidos de dolor 
(20) a casos de evolución fatal (21-23). Aunque algunos autores han encontrado una asociación con linfomas malignos, que puede llegar hasta el $15 \%(24,25)$, en otras series no se ha observado esta relación (3), por lo que la asociación puede ser casual.

Las opciones terapéuticas se basan en pequeñas series o casos clínicos aislados, dado que no existen estudios a doble ciego al respecto. El tratamiento quirúrgico queda reservado para los casos con obstrucción intestinal extrínseca (26). Los corticoesteroides $(7,9,27)$ y la combinación de corticoides con azatioprina (28) se han demostrado eficaces mejorando el curso de la enfermedad y evitando el paso a fibrosis. Se ha observado una buena respuesta a la ciclofosfamida en casos de mesenteritis esclerosante (29) y en descripciones previas de paniculitis de larga evolución (20,30), con mejoría clínica y desaparición de las lesiones mesentéricas. En nuestro caso, la gran afectación clínica de la paciente, nos decidió a instaurar el tratamiento inmunosupresor con ciclofosfamida y prednisona, y a diferencia de los casos previamente descritos, en los que se utilizó la vía oral, se optó por la vía endovenosa en forma de bolos mensuales, por su menor toxicidad en comparación con la administración diaria vía oral (31). Tras el tercer ciclo se

\section{Bibliografía}

1. Kipfer RE. Mesenteric Panniculitis and retractile mesenteritis. Haubrich WS, Schaffner F, Berk JE, editores. Bockus Gastroenterology. Philadelphia: WB Saunders Company 1995; 3106-3109.

2. Van der Hulst RW, Rauws EA, Tytgat GN. Mesenteritis secondary to the use os a pneumatic jackhammer. Eur J Gastroenterol Hepatol 1995; 6: 573-575.

3. Ogden WW, Bradburn DM, Rives JD. Panniculitis of the mesentery. Ann Surg 1960; 151: 659-665.

4. Kipfer RE, Moertel CG, Dahlin DC. Mesenteric lipodystrophy. Ann Intern Med 1974; 80: 582-588.

5. Emory TS, Moniham JM, Carr NJ, Sobin LH. Sclerosing mesenteritis, mesenteric panniculitis, and mesenteric lipodystrophy : a single entity. Am J Surg Pathol 1997; 21: 392-398.

6. Parra-Dávila E, Mckenney MG, Sleeman D, Hartmann R, Rao RK, McKenney K et al. Mesenteric Paniculitis: Case Report and Literature Review. Am Surg 1998; 64: 768-771.

7. De la Peña A, Yuste Ara JR, Beloqui Ruiz O, Prieto Valtueña J. La paniculitis mesentérica en el diagnóstico diferencial del dolor abdominal. Rev Esp Enferm Digest 1996; 88(7): 505-508.

8. Sans M, Varas M, Anglada A, Baschs ME, Navarro S, Brugués J. Mesenteric panniculitis presenting as fever of unknown origin. Am J Gastroenterol 1995; 90: 1159-1161.

9. Suriñach JM, Alegre J, Allende H, aleman C, Jufresa J, Fernández de Sevilla T. Severa pérdida de peso en el debut de una paniculitis mesentérica. An Med Interna (Madrid) 1995; 12: 597-599.

10. Honore LH. Partial obstruction of the small bowel caused by retractile mesenteritis. South Med J 1981; 74: 73-75.

11. Gironés Vila J, Alcobilla Ferrara E, Codina Barrera A, Gómez Castella F, Farres Coll R, Masvidal Calpe R, Fernández Gutiérrez F, Sant Masoliver F. Mesenteritis retráctil. A propósito de 3 casos. Rev. Esp Enf Digest 1995; 87 (1): 62-64.

12. Pérez-Fontán FJ, Soler R, Sánchez J, Iglesias P, Sanjurjo P, Ruiz J. Retractile mesenteritis involving the colon: barium enema, sonographic, and CT findings. Am J Roentgenol 1986; 147: 937-940.

13. Nikhal Patel, Samy F. Saaleb, Steven K Teplick General case of the day. Radiographics 1999; 19(4): 1083-1085.

14. Kopecky K, Lappas J, Baker M, Madura S. Mesenteric panniculitis: CT appearance. Gastrointestinal Radiol 1988; 13: 273-274.

15. Mata JM, Inaraja L, Martín J, Olazábal A, Castilla MT. CT features of mesenteric panniculitis. J Computer Assisted Tomography 1987; 11: 1021-1023.

16. Espinosa MD, Anguita F, Nogueras F, Castro T, De Teresa FJ, Martín Vivaldi R. Paniculitis mesentérica: diagnóstico laparoscópico. Rev Esp Enferm Digest 1997; 89 (10): 791-799.

17. Jiménez, FJ, Pina, L. Diagnóstico radiológico y confirmación histológica mediante biopsia percutánea. Radiología 1996; 38 (4): 276-279. evidenció una franca mejoría tanto clínica como la desaparición objetiva de las lesiones. El tratamiento fue bien tolerado y no hubo efectos secundarios de interés.

Es destacable en nuestro caso la afectación del colon que tan sólo se ha descrito en el 5\% de casos de paniculitis mesentérica en literatura inglesa (32), aunque en los casos japoneses alcanza el 54\% (33), y es muy poco frecuente que la paniculitis mesentérica afecte el retroperitoneo

Como conclusión, la paniculitis mesentérica puede asociarse con afectación retroperitoneal aunque es muy poco frecuente y en la revisión bibliográfica realizada no hemos encontrado otro caso en el que se produjera englobamiento de un uréter. Tampoco hemos hallado en la revisión bibliográfica, que con anterioridad a nuestro caso se hubiera utilizado la ciclofosfamida endovenosa. En casos similares al descrito con paniculitis mesentérica extensa y con clínica agresiva, el tratamiento inmunosupresor con ciclofosfamida endovenosa en pulsos y corticoides puede ser una buena opción terapéutica, dada la rápida resolución del caso presentado y la ausencia de efectos secundarios asociados.

18. Coffin CM, Watterson J, Priest JR, Dehner LP. Extrapulmonary inflammatory myofibroblastic tumor (inflammatory pseudotumor). A clinicopathologic and immunohistochemical study of 84 cases. Am J Surg Pathol 1995; 19: 859-872.

19. James K. Kelly. Idiopathic Retractile ( Sclerosing) Mesenteritis and Its Diferencial Diagnosis. Am J Surgical Pathol 1989; 13 (6): 513-521.

20. Pedro-Botet J, Ordi J. Varón de 36 años con dolor abdominal de 19 años de evolución y una masa en fosa ilíaca derecha. Med Clin ( Barc) 1996; 107: 628-635.

21. Caballero Oliver A. Paniculitis mesentérica: un caso de presentación aguda y ifatal? An Med Interna (Madrid) 1995; 12: 36-38.

22. Soergel KH, Hensely GT. Fatal mesenteric panniculitis. Gastroenterology 1966; 51: 529-536.

23. Andersen JA, Rasmussen NR, Pedersen JK. Mesenteric panniculitis: a fatal case. Am J Gastroenterol 1982; 77: 523-525.

24. Pérez-Ferriols A, Febrer-Bosch I, Oliver Martínez V, Rodríguez Serra M, Sabater Marco V, Aliaga Boniche A. Primary soft tissue lymphoma associated with mesenteric lipodystrophy. Am J Dermatopathol 1993; 15: 363-367.

25. Kipfer R, Moertel C, Dahlin D. Mesenteric lipodystrophy.Ann Intern Med 1974; 80: 582-588.

26. López L., Cuevas V, Rodríguez MA et al. Mesenteritis retráctil. Una entidad causante de obstrucción intestinal y malabsorción. Gastroenterol Hepatol 1983; 6: 537-539.

27. Kikiros C, Edis A. Mesenteric panniculitis resulting in bowel obstruction and response to steroids. Aust NZ J Surg 1989; 59: 287-290.

28. Tytgat GN, Roozendaal K, Winter W, Esseveld MR. Successfull treatment of a patient with retractile mesenteritis with prednisone and azathioprine. Gastroenterology 1980; 79: 352-356.

29. Bush RW. Sclerosing Mesenteritis. Response to Ciclophosphamide. Arch Intern Med 1986; 146: 503-505.

30. Trautwein C, Hermann E, Rambow A, Lohr H, Klose P, Gabbert H, Poralla T. Retractile mesenteritis. Diagnostic and therapeutic aspects. Dtsch Med Wochenschr 1990; 115(5): 174-178.

31. Haubitz M, Schellong S, Gobel U, Schurek HJ, Schaumann D, Koch KM, Brunkhorst R. Intravenous pulse administration of cyclophosphamide versus daily oral treatment in patients with antineutrophil cytoplasmic antibody-associated vasculitis and renal involvement: a prospective, randomized study. Arthritis Rheum 1998; 41(10): 183544.

32. Durst AL, Freund H, Rosenman E, Birnbaum D. Mesenteric panniculitis. Review of the literature and presentation of cases. Surgery 1977; 81: 203-211.

33. Adachi Y, Mori M, Enjoji M, Ueo H, Sugimachi K. Mesenteric panniculitis of the colon. Review of the literature and report of two cases. Dis Colon Rectum 1987; 30: 962-966. 